\title{
B-natriuretic peptide serum levels in neonates with persistent pulmonary hypertension
}

\author{
Tamer A. Abdel Hamid ${ }^{1 *}$, Zahraa Mohamed Ezz Eldin', Ranya Aly Hegazy ${ }^{1}$, Reem Ibrahim Esmail ${ }^{1}$ and \\ Lamis Mohsen Ahmed El-sholkamy²
}

\begin{abstract}
Background: Better management of persistent pulmonary hypertension of neonates (PPHN) required new markers that can predict the response of patient to treatment and thus influence the medical decision to avoid short-term and long-term adverse effects. Hence, we aimed to evaluate B-natriuretic peptide (BNP) serum levels in neonates with PPHN and to correlate its levels with disease severity and response to treatment.

Patients and methods: The study included 60 neonates (30 PPHN patients and 30 healthy subjects). BNP was assessed, using the ELISA technique, at admission and after 4 days of treatment.

Results: Initial serum BNP levels were significantly higher in patients compared to controls $(p<0.001)$. Patients with mild severity showed lower BNP levels compared to patients with moderate severity, while patients with moderate severity showed decreased levels in comparison to severe cases $(p<0.001)$. The group of patients who showed remission after 4 days of treatment had lower pre-treatment and post-treatment BNP levels, compared to patients who showed incomplete remission. Initial levels of BNP showed strong positive correlations with pulmonary artery pressure (PAP) and oxygenation index (OI) before treatment $(r=0.9, p<0.001$ and $r=0.85, p=0.001)$, while BNP after treatment showed moderate positive correlations with PAP before treatment $(r=0.6, p=0.001)$ and OI before treatment $(r=0.6, p=0.001)$. Analysis of the ROC curve revealed an AUC of 0.83 with $\mathrm{Cl}=0.7-0.98$ and $p=0.003$.

Conclusions: BNP may play a role in the pathogenesis of PPHN, and BNP serum level can be used as a marker to detect disease severity and predict response to treatment.
\end{abstract}

Keywords: B-natriuretic peptide, Pulmonary hypertension, Neonates, PPHN

\section{Background}

Persistent pulmonary hypertension of the newborn (PPHN) is considered one of the serious disorders in the neonatal period with prevalence 0.5 to 7 patients per 1000 live births [1]. A recent study reported a very high frequency among newborns in Egypt, which extends to $5 / 1000$ live births [2]. It is characterized by sustained high resistant pulmonary circulation after delivery and failure to shunt to a low resistant pulmonary circulation

\footnotetext{
* Correspondence: faragshayma@gmail.com

${ }^{1}$ Pediatric Department, Teaching Hospital of Cairo University, Cairo, Egypt Full list of author information is available at the end of the article
}

state [3]. The increased pulmonary vascular resistance will lead to low pulmonary blood flow, interfering with proper gas exchange in the lungs, leading to decreased blood oxygen level, severe respiratory distress, and high rate of morbidity and mortality $(4-33 \%)[1,4]$. Echocardiography is the gold standard to confirm the diagnosis of PPHN. The oxygenation index (OI) is commonly used during the management of PPHN as it is considered a better indicator of lung injury compared to the ratio between fraction of inspired oxygen concentration $\left(\mathrm{FiO}_{2}\right)$ and pressure of oxygen in the arterial blood $\left(\mathrm{PaO}_{2}\right)$ [5]. Despite the great achievement in the management of PPHN, the mortality rate caused by this illness is about 
$20 \%$ of affected neonates [6]. Moreover, the occurrence of prolonged hypoxemia may lead to adverse long-term sequelae such as chronic respiratory disorders and neurological developmental problems [7]. Prevention of prolonged hypoxemia depends on early diagnosis of PPHN. Particularly, this early diagnosis faces many challenges especially with the absence of a proper diagnostic method and the absence of specific clinical presentation in these patients as most of the neonatal diseases start with respiratory problems [8].

B-natriuretic peptide (BNP) is a natriuretic peptide hormone that is secreted by the ventricular myocardium in response to an increased stress of cardiac myocytes [9]. After birth, high pulmonary artery pressure (PAP) leads to secretion of large amount of BNP from stressed ventricles into the circulation. BNP acts as a potent vasodilator and diuretic mediator. Therefore, it can play a crucial role in hemodynamic adaptation after birth. Thus, the subsequent fall in PAP due to lung expansion, and the onset of diuresis with renal maturation explain the further fall in BNP levels [10]. In PPHN, production of BNP by the stressed myocardium increases. After being released into the circulation in large amount, BNP targets the intrinsic guanylyl cyclase activity leading to elevation of intracellular cyclic guanosine monophosphate (cGMP) that modulates and controls several cellular and physiological function including control of volume overload and cardiac filling pressure [11].

Accumulating data suggested its role as a biomarker of right ventricular dysfunction in patients with PPHN. However, there is scarcity of data about its role in PPHN among Egyptian neonates.

Our study aimed to evaluate BNP serum levels in neonates with PPHN and to correlate these levels with disease severity and response to treatment.

\section{Methods \\ Subjects}

This prospective case-control study was conducted on 30 full-term neonates, aged 1 day and diagnosed with PPHN. All cases were enrolled from the neonatal intensive care unit (NICU). The diagnosis of PPHN was suspected when the level of neonatal hypoxemia is disproportionate to the degree of respiratory distress and pulmonary parenchymal radiological findings. The diagnosis was further confirmed by echocardiographic findings that showed increased PAP, with demonstrable shunts across the patent ductus arteriosus (PDA) or patent foramen ovale (PFO) and the absence of cyanotic heart disease [12]. Before treatment, the severity of the disease was identified by the following criteria: (1) mild: pulmonary artery pressure (PAP) is $35-50 \mathrm{mmHg}$, (2) moderate: PAP is $51-70$
$\mathrm{mmHg}$, and (3) severe: PAP is above $70 \mathrm{mmHg}$ [13]. The included patients were subjected to treatment as scheduled by the protocol for the treatment of PPHN [2]. We followed up the patients till the age of day 4 . According to the response to treatment, patients were subdivided into two groups: the first group showed remission and required cessation of treatment (20 patients) and the other group did not show remission and required prolonged treatment or change in the treatment plan (10 patients).

Exclusion criteria were based on the presence of any of the following: (1) patients' mothers with any chronic disease, autoimmune disease, and cancer and (2) premature neonates, neonates with respiratory distress syndrome, sepsis, and chromosomal abnormalities, and neonates with congenital heart disease.

The study also included 30 age- and sex-matched healthy neonates who served as the control group. The included control subjects were full term with no respiratory or cardiac problems and have normal obstetric history for their mothers.

Informed consent was obtained from the parents of all neonates included in the study. The study was approved by the ethics committee in agreement with the Helsinki Declaration of 2015.

\section{Methods}

All studied patients were subjected to full history taking and full clinical examination. Routine laboratory markers were also done including complete blood picture, ESR, and C-reactive protein (CRP) to exclude sepsis.

Echocardiography was done for all patients using portable echocardiography "sonosite 180 plus" using probes $5-7 \mathrm{mHz}$ for assessment of PAP and chamber enlargement and functions. All measurements of the left ventricle, right ventricle, pulmonary artery, and its branches were assessed, and the presence of PDA and PFO were noted. The OI was calculated according to the following formula: $\mathrm{OI}=\mathrm{MAP}$ (in $\mathrm{cm} \mathrm{H}_{2} \mathrm{O}$ ) $\times$ $\mathrm{FiO}_{2} \times 100 / \mathrm{PaO}_{2}[14]$.

Assessment of B-natriuretic peptide serum level was done using a solid-phase sandwich ELISA technique, supplied by My BioSource, Inc, USA (catalog number MBS731384). Two samples were collected from each patient, one at day 1 before initiation of therapy and the other at day 4 of life after initiation of therapy. All samples were run in duplicate to validate the results.

\section{Statistical methods}

Data were recorded and entered using the SPSS version 25 . Data was summarized using mean \pm standard deviation (mean $\pm \mathrm{SD}$ ) or median (interquartile range) (IQR) 
for parametric or non-parametric distributed quantitative variables, respectively, and frequencies (number of cases) for categorical variables. Comparisons between cases and controls were done using unpaired Student $t$ test, while comparison between values measured before and after treatment was done using paired $t$ test. For comparing categorical data, a chisquare $\left(x^{2}\right)$ test was performed and Fisher's exact test was used instead of it only when the expected frequency is less than 5. Correlations between quantitative variables were done using Pearson's correlation coefficient. Univariate logistic regression analysis was done to evaluate pre-treatment BNP serum levels as a risk factor and predictor for PPHN after the adjustment of associated covariates. A receiver operating curve (ROC) was constructed, and area under the curve (AUC) and 95\% confidence interval (CI) were reported to test the ability of BNP serum level to diagnose and predict outcome of the PPHN. A $p$ value $<0.05$ was considered statistically significant.

\section{Results}

This study was conducted on 30 newborns with PPHN. The study also included 30 full-term neonates who served as the control group. All patients and controls were full term with a gestational age of mean $37.9 \pm 0.78$ and $37.8 \pm 0.6$ weeks $(p=0.6)$. All subjects included in the study were at day 1 after delivery. Both patients and controls were matched for birth weight (grams) (2960 \pm 190, $3020 \pm 148, p=0.2$ ), gender (21 males, 12 males, $p$ $=0.06)$, mode of delivery (18 vaginal, 22 cesarean section, $p=0.4)$, and age of their mothers $(27 \pm 3.2,28$ \pm 3.1 years, $p=0.3$ ). All controls had normal PAP measurements with median 29 and range $25-30 \mathrm{mmHg}$, while all patients had pulmonary hypertension with median of PAP 54 and range between 44 and $84 \mathrm{mmHg}$. The patients were divided according to the severity of the disease into mild (11 patients), moderate (14 patients), and severe (5 patients) groups. The characteristics of each group are presented in Table 1.

Serum BNP levels were significantly higher in patients before administration of treatment compared to controls (Fig. 1, $p<0.001$ ). Regarding patient groups, before administration of treatment, mild cases showed lower BNP serum levels compared to moderate cases, while moderate cases showed decreased levels in comparison to severe cases $(p<0.001$ for all comparisons) (Fig. 1). After 4 days of administration of treatment, a non-parametric paired test showed that BNP levels statistically decreased in each group (mild, moderate, and severe) compared to its levels within these groups before treatment $(p=0.001,<0.001$, and 0.04 , respectively) (Table 1 ).

Moreover, patients who experienced remission and those who did not experience remission after treatment showed a significant reduction of post-treatment BNP serum levels compared to pre-treatment levels. However, the group of patients who showed remission had lower pre-treatment and post-treatment BNP serum levels compared to patients who did not show complete remission (Fig. 2).

Pearson's correlation analysis revealed that serum levels of BNP before treatment showed strong positive correlations with values of PAP and OI before treatment $(r=0.9, p<0.001$ and $r=0.85, p=0.001)$ (Fig. 3) and moderate positive correlation with values of PAP and OI

Table 1 Characteristics of patients' groups

\begin{tabular}{|c|c|c|c|c|}
\hline Variable & Mild (11 patients) & Moderate (14 patients) & Severe (5 patients) & $p$-value \\
\hline Gestational age (weeks) ${ }^{a}$ & $38.2 \pm 0.8$ & $37.7 \pm 0.7$ & $37.7 \pm 0.5$ & 0.6 \\
\hline Birth weight $(\text { grams })^{a}$ & $2904 \pm 170$ & $2932 \pm 200$ & $2893 \pm 150$ & 0.3 \\
\hline Gender (male/female) ${ }^{b}$ & $7 / 4$ & $10 / 5$ & $4 / 0$ & 0.2 \\
\hline Mode of delivery (vaginal/cesarean section) ${ }^{b}$ & $7 / 4$ & $8 / 6$ & $3 / 2$ & 0.7 \\
\hline Age of mothers (years) ${ }^{a}$ & $24.1 \pm 3.2$ & $23.8 \pm 2.9$ & $23.4 \pm 4.1$ & 0.5 \\
\hline Pulmonary artery pressure $(\mathrm{mm} \mathrm{Hg})^{c}$ & $48(45-50)$ & $55(53-57)$ & $65(64-79)$ & 0.001 \\
\hline Oxygenation index $(\mathrm{OI})^{c}$ & $10.8(9.6-12.1)$ & $19.7(16.9-22.3)$ & $31.7(27.9-36.2)$ & 0.001 \\
\hline \multicolumn{5}{|l|}{ Response after 4 days of treatment ${ }^{b}$} \\
\hline Remission/no remission & $9 / 2$ & $9 / 5$ & $2 / 3$ & 0.1 \\
\hline \multicolumn{5}{|l|}{ B-natriuretic peptide serum level $(\mathrm{pg} / \mathrm{ml})^{\mathrm{C}}$} \\
\hline Before treatment & $310(305-330)$ & $350(340-350)$ & 465 (450-495) & 0.001 \\
\hline After treatment & 90 (88-96) & 97 (94-99) & $105(98-110)$ & 0.001 \\
\hline
\end{tabular}

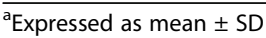

bexpressed as frequency

cexpressed as median (IQR) 


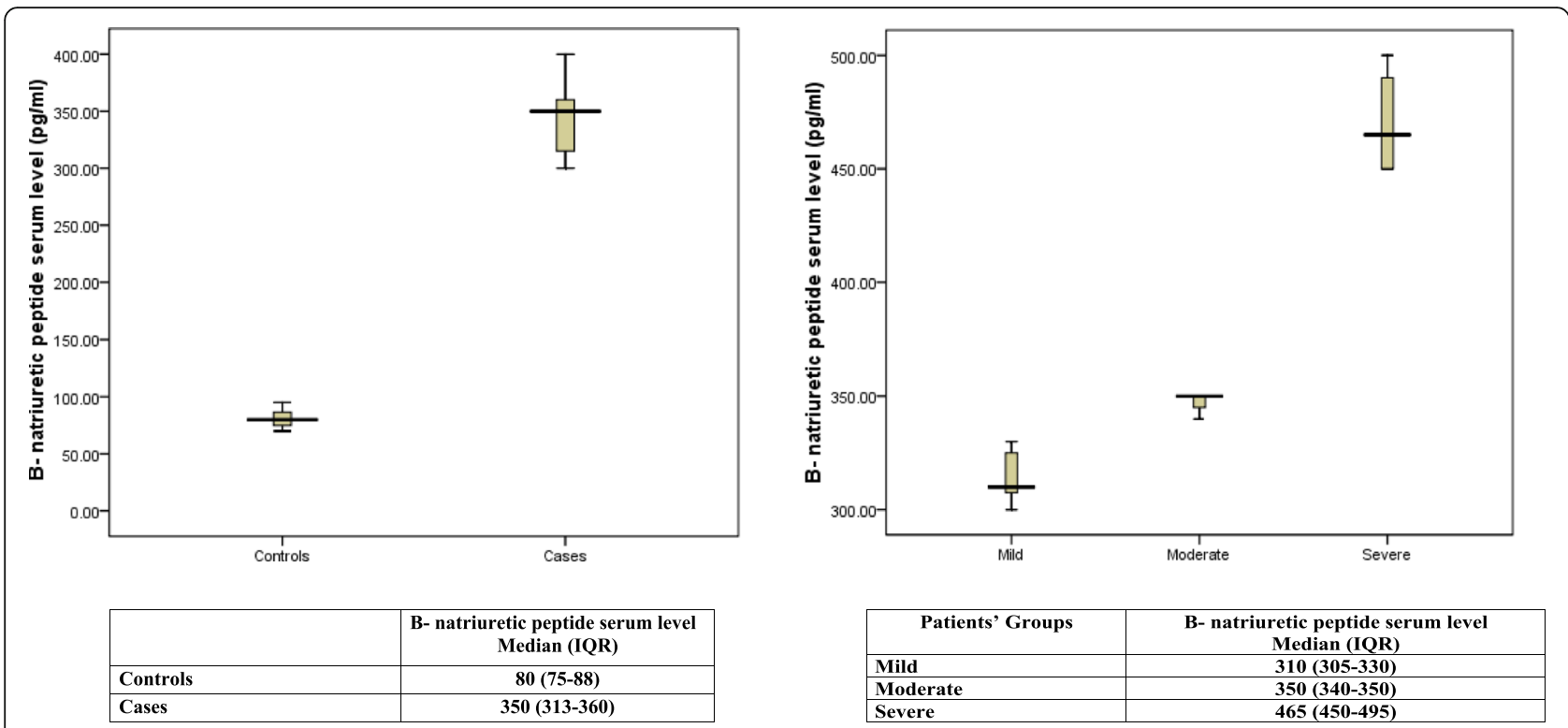

Fig. 1 Comparisons of serum B-natriuretic peptide (BNP) levels among patients and controls. a Serum BNP levels were significantly higher in patients before administration of treatment compared to controls. b Cases that showed mild disease severity had lower BNP serum levels compared to moderate patients, while moderate patients showed decreased levels in comparison to severe patients

after treatment $(r=0.64, p=0.001$ and $r=0.63, p=$ 0.001) (Fig. 3). Moreover, BNP after treatment showed moderate positive correlations with post-treatment values of PAP and OI $(r=0.59, p=0.001$ and $r=0.58, p$ $=0.001$ ) (Fig. 3).

Univariate logistic regression analysis was performed to confirm that BNP pre-treatment serum level could act as an independent risk factor and predictor for treatment response for PPHN after the adjustment of gender, gestational age, and weight and degree of severity of the disease. This analysis confirmed the association between BNP pre-treatment serum level with the risk of PPHN development $(p=0.02$, odds ratio $=2.1, \mathrm{CI}=0.9-1.3)$ and with the response to treatment $(p=0.01$, odds ratio $=1.9, \mathrm{CI}=1-1.17$ ).

As BNP pre-treatment serum level was a significant laboratory indicator and predictor for PPHN in univariate analysis, we used it to plot the ROC curve that revealed an AUC of 0.83 with $\mathrm{CI}=0.7-0.98$ and $p=0.003$ (Fig. 4). Our data showed that pretreatment BNP serum level of $197 \mathrm{pg} / \mathrm{ml}$ has a specificity of $95 \%$ and a sensitivity of $100 \%$ in predicting PPHN and a level of $>302 \mathrm{pg} / \mathrm{ml}$ has a specificity of $90 \%$ and a sensitivity of $100 \%$ in predicting treatment response.

\section{Discussion}

PPHN is a serious medical condition with a high rate of morbidity and mortality. Thus, it should be managed as soon as possible to avoid prolonged hypoxia that causes short-term and long-term morbidities [15]. Although initial echocardiographic assessment remains necessary for the diagnosis of $\mathrm{PPNH}$, further markers to influence the management and follow-up of patients during treatment are required.

BNP is secreted by cardiac ventricles in response to ventricular filling pressure to stabilize blood volume and regulate blood pressure, so its serum level may provide information on ventricular function. Our results showed that BNP is markedly increased in PPHN patients compared to controls and this finding also supports that BNP may play a role in the pathogenesis of PPHN that was reported before by different studies [16-18]. Altered levels of BNP were also reported in various neonatal disorders, and Reynolds et al. reported that BNP has the capability to differentiate respiratory distress syndrome from PPHN and to differentiate between patients with hemodynamic or cardiac compromise and those with a purely respiratory condition [16]. BNP was also suggested as a screening tool for the presence of PDA in preterm neonates as BNP level was reported to be high in the presence of significantly large PDA and this level correlated well with ductal size [19, 20]. BNP was also studied in patients with congenital heart disease and showed higher levels in these patients including the clinically stable ones compared to healthy subjects [21]. 


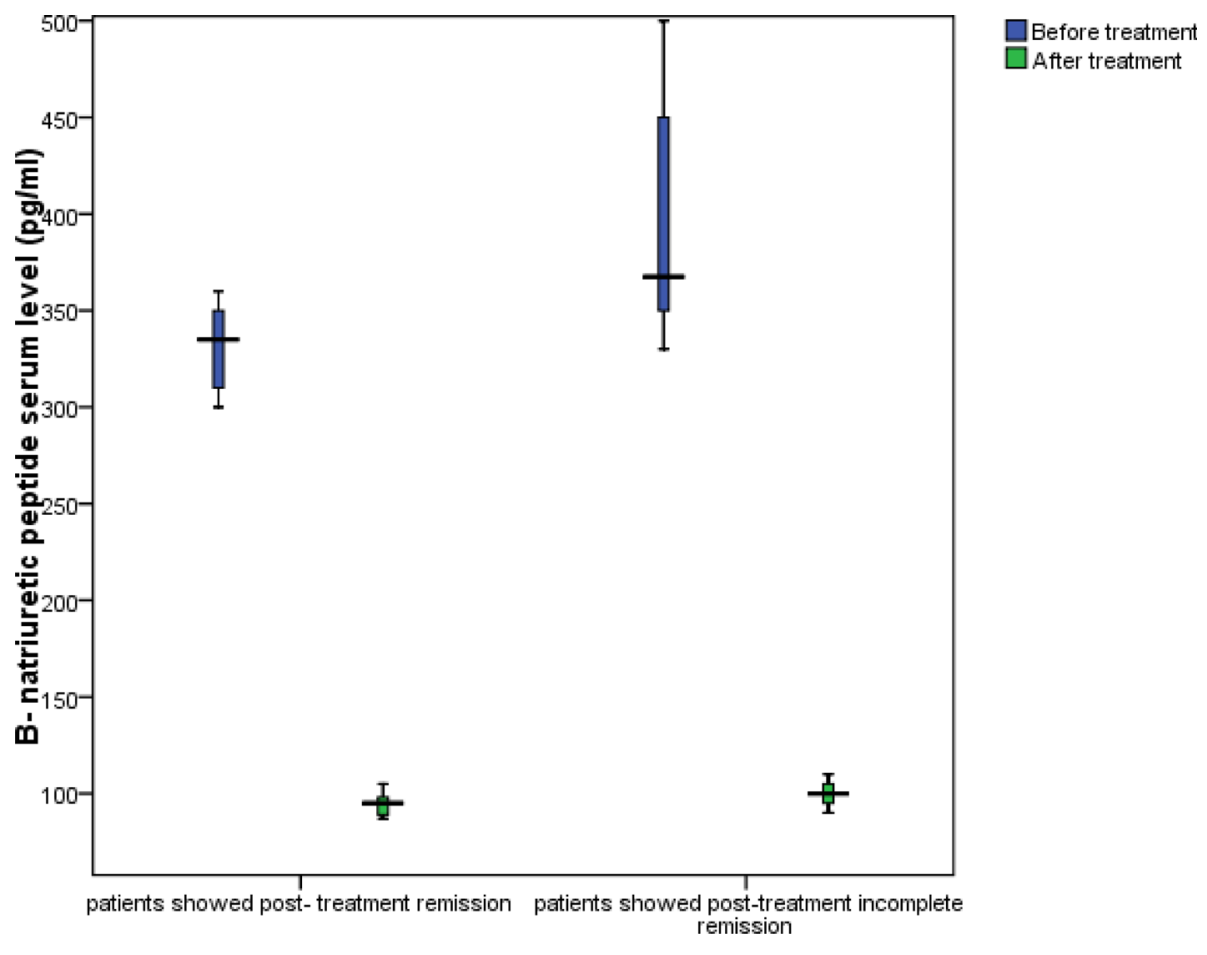

\begin{tabular}{|l|c|c|c|}
\hline $\begin{array}{c}\text { B-natriuretic peptide } \\
\text { Median (IQR) }(\mathrm{pg} / \mathrm{ml})\end{array}$ & $\begin{array}{c}\text { Patients showed complete } \\
\text { remission after treatment }\end{array}$ & $\begin{array}{c}\text { Patients showed incomplete } \\
\text { remission after treatment }\end{array}$ & p value \\
\hline -Before treatment & $\mathbf{3 3 5 ( 3 1 0 - 3 5 0 )}$ & $\mathbf{3 7 6 ( 3 5 0 - 4 5 7 )}$ & $\mathbf{0 . 0 0 1}$ \\
-After treatment & $\mathbf{9 5 ( 8 8 - 9 8 )}$ & $\mathbf{1 0 0 ( 9 4 - 1 0 6 )}$ & $\mathbf{0 . 0 0 5}$ \\
p value & $<0.001$ & $<0.001$ & \\
\hline
\end{tabular}

Fig. 2 Comparisons of B-natriuretic peptide (BNP) serum levels among patients that showed complete remission after treatment and those who did not show complete post-treatment remission. Both patients who experienced remission and those who did not experience remission after treatment showed a significant reduction of post-treatment BNP serum levels compared to pre-treatment levels. However, the group of patients who showed remission had lower pre-treatment and post-treatment BNP serum levels, compared to patients who did not show complete remission

Moreover, to validate the association of BNP serum levels with disease severity, we subdivide the patients according to PAP measurements into mild, moderate, and severe cases. Therefore, we found that mild cases showed lower BNP serum levels compared to moderate cases, while moderate cases showed decreased levels in comparison to severe cases. Moreover, a strong positive correlation was detected between BNP pre-treatment level and both PAP and OI, suggesting the usefulness of the utility of BNP serum levels to assess the severity of PPHN. These findings were in agreement with others [1618]. Furthermore, patients who showed complete remission after a course of scheduled treatment also showed a marked decrease in BNP serum levels compared to patients who showed no remission.
Logistic regression and ROC analysis also confirmed the utility of pre-treatment BNP serum level as a predictor for response to treatment in PPHN. In agreement with our findings, Reynolds and his colleagues studied plasma BNP levels in three groups of neonates (1st group with PPHN, 2nd group with respiratory distress without PPHN, and 3rd group without respiratory distress) and reported that BNP levels elevated in the group with PPHN compared with the other two groups and remained significantly higher during the first 4 days of life [16]. Vijlbrief et al. conducted a study on 21 neonates with PPHN and found that BNP is elevated in these neonates suggesting its role as a biomarker for cardiac ventricular strain [9]. Moreover, they evaluated BNP levels in patients who showed rebound PPHN after 


\section{A}

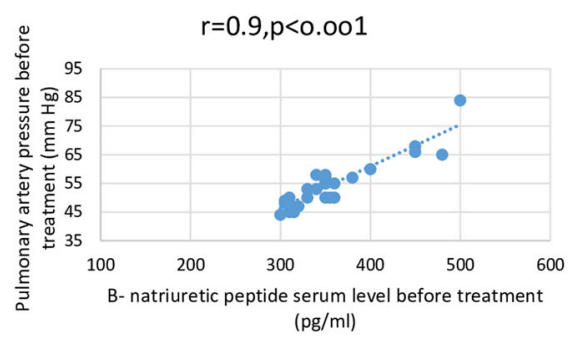

C

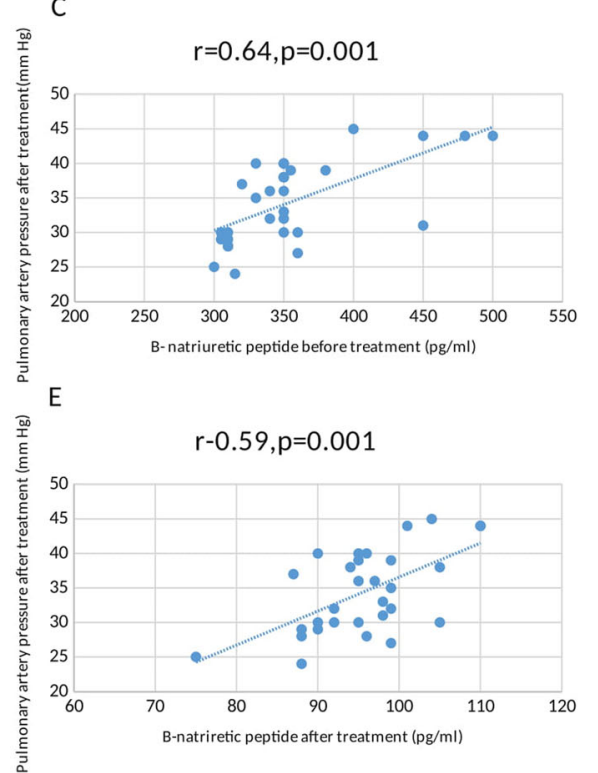

\section{B}

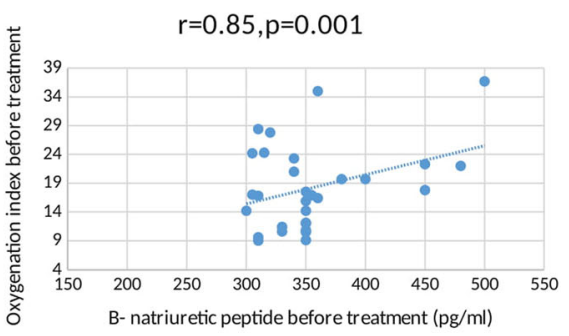

D
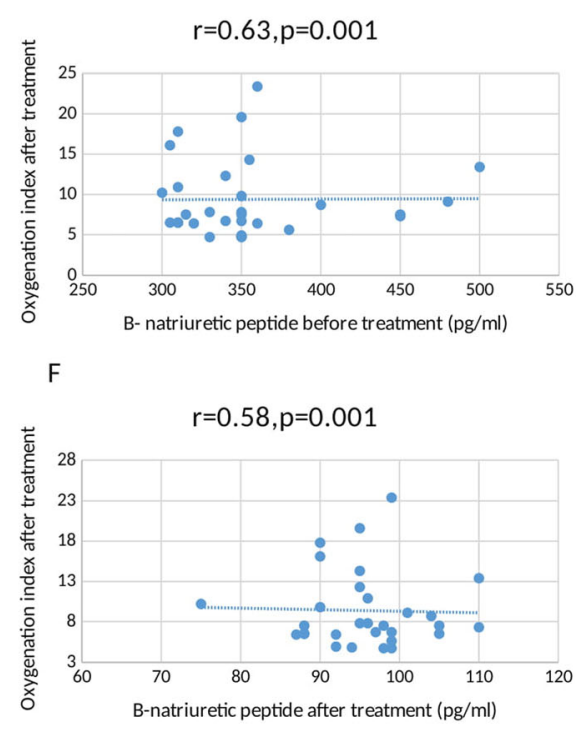

Fig. 3 Correlates of B-natriuretic peptide (BNP) with pulmonary artery pressure (PAP) and oxygenation index (OI). Pearson's correlation analysis revealed that serum levels of BNP before treatment showed strong positive correlations with values of PAP and OI before treatment $(\mathbf{a}, \mathbf{b})$ and moderate positive correlation with values of PAP and Ol after treatment (c, d). Moreover, BNP after treatment showed moderate positive correlations with post-treatment values of PAP and OI $(\mathbf{e}, \mathbf{f})$

iNOS treatment and concluded that BNP is a useful marker in the evaluation of the course of treatment in PPHN patients and prediction of rebound PPHN [9]. Shan and his colleagues demonstrated that neonates with PPHN and whom required iNO in their treatment had higher initial BNP levels compared with others who did not require iNO in their treatment, and BNP had 100\% sensitivity to predict the need for iNO in neonates with PPHN [17]. Our data also showed that the initial BNP serum level of 197 $\mathrm{pg} / \mathrm{ml}$ showed a specificity of $95 \%$ and a sensitivity of $100 \%$ in predicting PPHN and a level of $>302 \mathrm{pg} /$ $\mathrm{ml}$ has a specificity of $90 \%$ and a sensitivity of $100 \%$ in predicting treatment response for PPHN. Reynolds et al. also demonstrated that an initial BNP level of $>159 \mathrm{pmol} / \mathrm{l}$ has a specificity of $90 \%$ and a sensitivity of $100 \%$ in predicting PPHN [16]. In a retrospective cohort analysis, Cuna et al. reported that a BNP level of $220 \mathrm{pg} / \mathrm{ml}$ showed a sensitivity of $90 \%$ and a specificity of $65 \%$ for predicting outcome in preterm infants with pulmonary hypertension [22]. Baysal and his colleagues mentioned that the ROC for plasma BNP value at $125.5 \mathrm{pg} / \mathrm{mL}$ showed an AUC of $91 \%$ with a sensitivity of $88.9 \%$ and a specificity of $96.9 \%$ for predicting low cardiac output [23]. The differences of values mentioned in the previous studies may be due to the differences in study design and inclusion and exclusion criteria. The utility of BNP as a marker of disease severity has been reported in other diseases including bronchopulmonary dysplasia in preterm infants and pulmonary hypertension in older infants [24, 25]. Interestingly, recent researches have been done to suggest the possible use of BNP as a therapeutic agent in PPHN, but based on our knowledge, no trials in neonates have been reported until now [26, 27]. 


\section{$\mathrm{AUC}=0.83, \mathrm{Cl}=0.7-0.98 \cdot \mathrm{P}=0.003$}

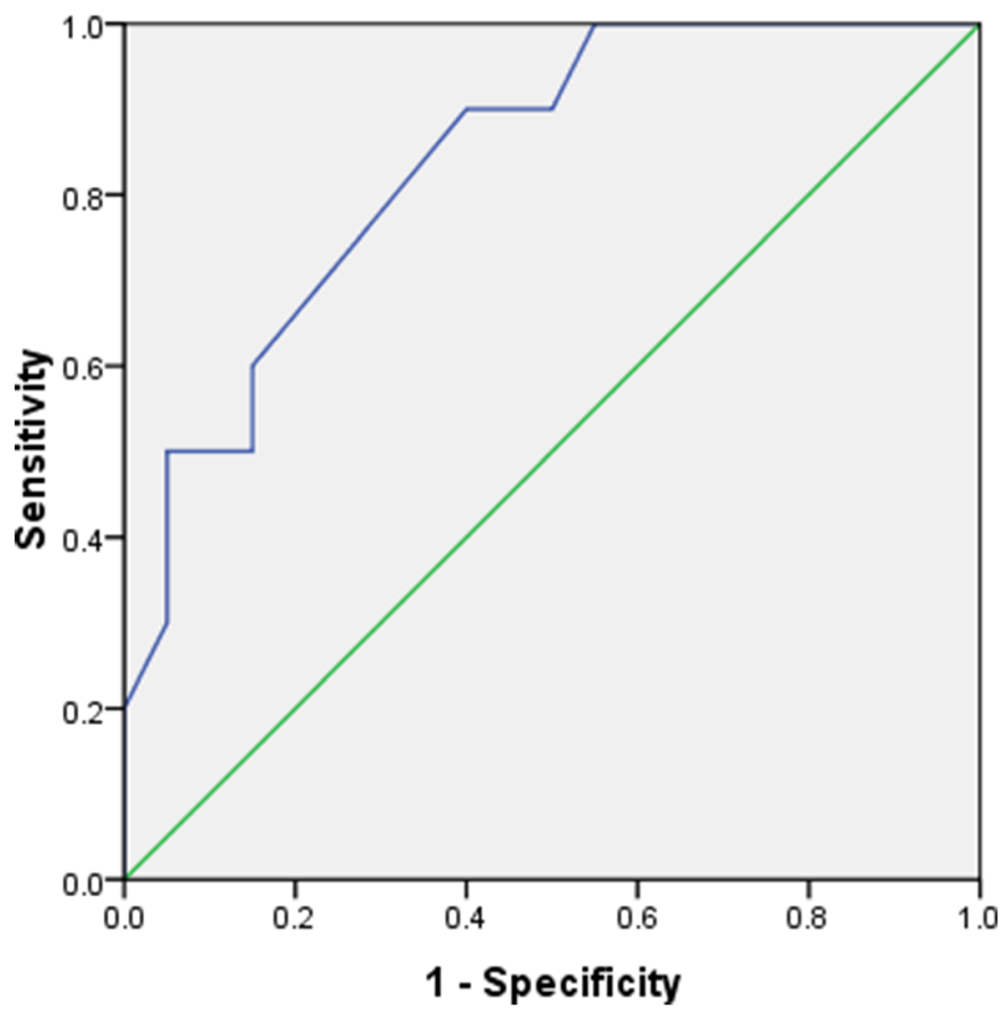

Fig. 4 ROC curve of pre-treatment of B-natriuretic peptide (BNP) serum levels. ROC analysis of the initial BNP serum level that revealed an AUC of 0.83 with $\mathrm{Cl}=0.7-0.98$ and $p=0.003$

\section{Conclusion}

We concluded that BNP may play a role in the pathogenesis of PPHN and BNP serum level can be used as a marker to detect disease severity and predict response to treatment. Future studies are recommended with larger sample sizes to validate BNP as a marker for prognosis in PPHN and to identify its possible use as a therapeutic agent.

\section{Abbreviations}

PPHN: Persistent pulmonary hypertension of the newborn; BNP: B-natriuretic peptide; NICU: Neonatal intensive care unit; PAP: Pulmonary artery pressure; OI: Oxygenation index; IQR: Interquartile range; AUC: Area under the curve

\section{Acknowledgements}

The authors like to acknowledge all nursery staff of NICU.

\section{Authors' contributions}

All authors have read and approved the manuscript. TA contributed to the conception, design of the work, examination of patients, and interpretation of data; wrote the manuscript; and revised it. ZME contributed to the conception and design of the work and revised and approved the submitted version. RAH contributed to the conception, design of the work, and interpretation of the data and revised and approved the submitted version. RIE contributed to the conception and interpretation of the data, approved the submitted version, and agreed to be personally accountable for the author's own contributions. LME contributed to the conception, design of the work, and collection and interpretation of the data and revised and approved the submitted version

\section{Funding}

This work received no special fund.

\section{Availability of data and materials}

The datasets analyzed during the current study are available from the corresponding author on reasonable request.

\section{Declarations}

Ethics approval and consent to participate

Informed verbal consent was obtained from the parents of all neonates included in the study because most of the parents are illiterate. The study was approved by the ethics committee of Cairo University in agreement with the Helsinki Declaration of 2015.

\section{Consent for publication}

Not applicable

\section{Competing interests}

The authors declare that they have no competing interests.

\section{Author details}

${ }^{1}$ Pediatric Department, Teaching Hospital of Cairo University, Cairo, Egypt.

${ }^{2}$ Mounira General Hospital, Cairo, Egypt. 
Received: 7 August 2020 Accepted: 11 March 2021

Published online: 19 April 2021

\section{References}

1. El-Khuffash A, McNamara PJ, Breatnach C et al (2018) The use of milrinone in neonates with persistent pulmonary hypertension of the newborn - a randomised controlled trial pilot study (MINT 1): study protocol and review of literature. Matern Health Neonatol Perinatol 4:24. https://doi.org/10.1186/ s40748-018-0093-1

2. Abdel Mohsen AH, Amin ASJ (2013) Risk factors and outcomes of persistent pulmonary hypertension of the newborn in neonatal intensive care unit of Al-Minya University Hospital in Egypt. Clin Neonatol 2(2):78-82. https://doi. org/10.4103/2249-4847.116406

3. Lai MY, Chu SM, Lakshminrusimha S, Lin HC (2018) Beyond the inhaled nitric oxide in persistent pulmonary hypertension of the newborn. Pediatr Neonatol 59(1):15-23. https://doi.org/10.1016/j.pedneo.2016.09.011

4. Wakeel MAE, Sabry RN, El-Kassas GM et al (2019) Pentraxin 3: a potential novel predictor for neonatal pulmonary hypertension. Open Access Maced J Med Sci 7(15):2424-2427. https://doi.org/10.3889/oamjms.2019.638

5. Rawat M, Chandrasekharan PK, Williams A, Gugino S, Koenigsknecht C, Swartz D, Ma CX, Mathew B, Nair J, Lakshminrusimha S (2015) Oxygen saturation index and severity of hypoxic respiratory failure. Neonatology 107(3):161-166. https://doi.org/10.1159/000369774

6. Tang Z, Jiang M, Ou-Yang Z, Wu H, Dong S, Hei M (2019) High mobility group box 1 protein (HMGB1) as biomarker in hypoxia-induced persistent pulmonary hypertension of the newborn: a clinical and in vivo pilot study. Int J Med Sci 16(8):1123-1131. https://doi.org/10.7150/ijms.34344

7. Nair J, Lakshminrusimha S (2014) Update on PPHN: mechanisms and treatment. Semin Perinatol 38(2):78-91. https://doi.org/10.1053/j.semperi.2 013.11 .004

8. Brunner-La Rocca HP, Sanders-van Wijk S (2019) Natriuretic peptides in chronic heart failure. Card Fail Rev 5(1):44-49. https://doi.org/10.15420/cfr.2 018.26.

9. Vijlbrief DC, Benders MJ, Kemperman H, van Bel F, de Vries WB (2012) Btype natriuretic peptide and rebound during treatment for persistent pulmonary hypertension. J Pediatr 160(1):111-5.e1

10. Mannarino S, Garofoli F, Mongini E, Cerbo RM, Codazzi AC, Tzialla C, Mazzucchelli I, Perotti G, Tinelli C, de Silvestri A, Manzoni P, Stronati M (2010) BNP concentrations and cardiovascular adaptation in preterm and full term newborn infants. Early Hum Dev 86(5):295-298. https://doi.org/10.1 016/j.earlhumdev.2010.04.003

11. Takahashi M, Takeda S, Kurokawa S, Kubo T, Fukuda N, Izumi T (2003) Cyclic GMP production by ANP, BNP, and NO during worsening and improvement of chronic heart failure. Jpn Heart J 44(5):713-724. https://doi.org/10.1536/ jhj.44.713

12. Mohamed WA, Ismail M (2012) A randomized, double-blind, placebocontrolled, prospective study of bosentan for the treatment of persistent pulmonary hypertension of the newborn. J Perinatol 32(8):608-613. https:// doi.org/10.1038/jp.2011.157

13. Fu L, Zhang X (2019) Correlation between changes in brain natriuretic peptide and echocardiographic features in persistent pulmonary hypertension of newborn. J Matern Fetal Neonatal Med 33:1-5

14. Baquero H, Soliz A, Neira F, Venegas ME, Sola A (2006) Oral sildenafil in infants with persistent pulmonary hypertension of the newborn: a pilot randomized blinded study. Pediatrics. 117(4):1077-1083. https://doi.org/10.1 542/peds.2005-0523

15. Konduri GG, Kim OU (2009) Advances in the diagnosis and management of persistent pulmonary hypertension of the newborn (PPHN). Pediatr Clin N Am 56(3):579

16. Reynolds EW, Ellington JG, Vranicar M, Bada HS (2004) Brain-type natriuretic peptide in the diagnosis and management of persistent pulmonary hypertension of the newborn. Pediatrics 114(5):1297-1304. https://doi.org/1 0.1542/peds.2004-0525

17. Shah N, Natarajan G, Aggarwal S (2015) B-type natriuretic peptide: biomarker of persistent pulmonary hypertension of the newborn? Am J Perinatol 32(11):1045-1049. https://doi.org/10.1055/s-0035-1548540

18. Abdel Latif MT, Hammad BS, Mikhael NL, Elmirahem ME (2017) Role of brain-type natriuretic peptide in rapid diagnosis and prognosis of persistent pulmonary hypertension of the newborn. Alex J Pediatr 30(1):11-16

19. Flynn PA, da Graca RL, Auld PA, Nesin M, Kleinman CS (2005) The use of a bedside assay for plasma B-type natriuretic peptide as a biomarker in the management of patent ductus arteriosus in premature neonates. J Pediatr 147(1):38-42. https://doi.org/10.1016/j.jpeds.2005.03.040

20. Kim JS, Shim EJ (2012) B-type natriuretic peptide assay for the diagnosis and prognosis of patent ductus arteriosus in preterm infants. Korean Circ J 42(3): 192-196. https://doi.org/10.4070/kcj.2012.42.3.192

21. Nasser N, Perles Z, Rein AJ et al (2006) NT-pro BNP as a marker for persistent cardiac disease in children with history of dilated cardiomyopathy and myocarditis. Pediatr Cardiol 27(1):87-90. https://doi.org/10.1007/s00246005-1027-z

22. Cuna A, Kandasamy J, Sims B (2014) B-type natriuretic peptide and mortality in extremely low birth weight infants with pulmonary hypertension: a retrospective cohort analysis. BMC Pediatr 14(1):68. https://doi.org/10.11 86/1471-2431-14-68

23. Baysal A, Saşmazel A, Yildirim A, Ozyaprak B, Gundogus N, Kocak T (2014) O valor preditivo do peptídeo natriurético tipo-B em resultados de crianças com hipertensão pulmonar submetidas à cirurgia cardíaca congênita [The predictive value of plasma B-type natriuretic peptide levels on outcome in children with pulmonary hypertension undergoing congenital heart surgery]. Rev Bras Anestesiol 64(5):326-334. https://doi.org/10.1016/j.bjan.2 013.10.004

24. Bernus A, Wagner BD, Accurso F, Doran A, Kaess H, Ivy DD (2009) Brain natriuretic peptide levels in managing pediatric patients with pulmonary arterial hypertension. Chest. 135(3):745-751. https://doi.org/10.1378/chest. 08-0187

25. Xiong T, Kulkarni M, Gokulakrishnan G, Shivanna B, Pammi M (2020) Natriuretic peptides in bronchopulmonary dysplasia: a systematic review. J Perinatol 40(4):607-615. https://doi.org/10.1038/s41372-019-0588-2

26. El-Khuffash A, Molloy EJ (2007) Are B-type natriuretic peptide (BNP) and Nterminal-pro-BNP useful in neonates? Arch Dis Child Fetal Neonatal Ed 92(4): F320-F324. https://doi.org/10.1136/adc.2006.106039

27. Reynolds E, Conely ET, Vranicar M (2007) Nesiritide for the treatment of pulmonary hypertension and cor pulmonale in an infant. Pediatr Cardiol 28(3):229-233. https://doi.org/10.1007/s00246-006-0064-6

\section{Publisher's Note}

Springer Nature remains neutral with regard to jurisdictional claims in published maps and institutional affiliations.

\section{Submit your manuscript to a SpringerOpen ${ }^{\circ}$ journal and benefit from:}

- Convenient online submission

- Rigorous peer review

- Open access: articles freely available online

High visibility within the field

- Retaining the copyright to your article

Submit your next manuscript at $>$ springeropen.com 